LPTHE-02-01, hep-th/0201257

\title{
Comments on the topological open membrane
}

\author{
B. Pioline \\ LPTHE, Universités Paris VI et VII, 4 pl Jussieu, \\ 75252 Paris cedex 05, France
}

\begin{abstract}
Just as non-commutative gauge theories arise from quantising open strings in a large magnetic field, non-Abelian two-form gauge theories may conceivably be constructed by quantising open membranes in a large three-form magnetic background. We make some observations that arise in following this strategy, with an emphasis on the relation to the quantisation of volume-preserving diffeomorphisms (vpd). In particular, we construct consistent non-Abelian interactions of a two-form in $3+1$ dimensions, based on gauge invariance under vpd.
\end{abstract}

PACS numbers: 11.10.Kk, 11.10.Lm, 11.25.-w, 47.37.+q

Keywords: Non-commutative geometry, Nambu bracket, Non-Abelian tensor, Superfluid Helium

Of the many mysteries surrounding the theory formerly known as string theory, the dynamics of the five-brane is perhaps the most intriguing. While a single type IIA or M5 brane supports a free self-dual two-form gauge field on its world-volume, together with five transverse scalar degrees of freedom and their fermionic partners [1], the case of $N$ five-branes at small separation is much less understood. Analogy with the case of coinciding D-branes and duality with ALE singularities in type IIB string theory suggest that the chiral multiplet now transforms in the adjoint representation of a spontaneously broken $U(N)$, with the open membranes stretched between two five-branes playing the rôle of stringy $W$ bosons on the five-brane worldvolume [2]. This picture is at best suggestive however, since the quantization of the membrane, if at all sensible, is still beyond reach (see 梱 for a review of recent attempts however). Disregarding the fundamental origin of these low-energy degrees of freedom, no consistent local theory of interacting non-Abelian selfdual two-forms has been found to date, in line with no go theorems [5]. The nature of the $U(N)$ non-Abelian symmetry itself is unclear, since anomaly and entropy considerations hint at $N^{3}$ degrees of freedom rather than $N^{2}$ as expected on a naive perturbative basis [6 - an admittedly unwarranted expectation due to the absence of a tunable coupling.

On the other hand, recent progress in the understanding of D-branes in background fields has taught us that non-Abelian dynamics arise even at $N=1$, in the presence of a strong background magnetic or electric field [7]. Indeed, the effects of the higher-derivative Born-Infeld couplings can be resummed by going to a non-commutative description, where the $U(1)$ gauge invariance $\delta a_{\mu}=\partial_{\mu} \lambda$ is replaced by a transformation $\delta a_{\mu}=\partial_{\mu} \lambda+a_{\mu} * \lambda-\lambda * a_{\mu}$ formally identical to the usual Yang-Mills gauge invariance [8]. The Moyal deformation of the ordinary commutative product can in fact be derived without detailed knowledge of the dynamics of open strings in a magnetic background $B=d A$ : in the limit of large $B$, one may retain only the topological coupling $\int_{\Sigma} B$ from the action of the string with worldsheet $\Sigma$ (we omit the pull-back from target space). This in turn reduces to a coupling $\oint_{\gamma} A$ on the boundary, hence to a topological quantum mechanical model. For constant background, the action $\oint B_{i j} X^{i}(\tau)\left[d X^{j} / d \tau\right] d \tau$ is Gaussian and first order in time derivatives, and yields the Moyal product structure for the correlators $\left\langle\prod_{i=1 \ldots p} f_{i}\left(X\left(\tau_{i}\right)\right)\right\rangle=\int d^{n} x f_{1} * \cdots * f_{p}(x)$. To leading order, the effect of the strong magnetic field is therefore to dress the free Maxwell action with Moyal products [8].

From this point of view, it is very tempting to try and apply the same logic to the M5-brane. In the presence of a strong magnetic three-form field strength $H$, the selfduality equations become highly non-linear [9], and it is conceivable that these higher-derivative effects may be resummed in terms of a non-commutative deformation of the linear self-duality equations [37], hopefully provided by the quantization of the topological open membrane. If one can furthermore find a matrix realization formally isomorphic to this non-commutative deformation, one will have succeeded in producing a non-Abelian extension - hopefully the only one - of the free tensor dynamics. In this note, we present some observations that arose in following this strategy, in the hope that they will serve in attaining this goal. Our attempt at quantizing the topological open membrane is not by any means the first, see e.g. [11, 12, 13, 14, 15, however we hope to provide a different perspective on this still unsolved problem. Our salient result is a consistent deformation of the dynamics of an Abelian two-form in 3+1 dimensions, which uses the group of volume-preserving diffeomorphisms in 3 dimensions as a gauge group, and which will be presented in the last section. The main message we want to convey is that the non-Abelian dynamics of tensor multiplets and the quantization of the open membrane are closely connected to the quantization of the volume-preserving diffeomorphisms in 3 dimensions, or rather to its realisation by means of the Nambu bracket.

Topological open membranes. By analogy with the string, let us consider the dynamics of membranes in the 
background of a strong three-form field $H$. The coupling of a supermembrane to the background field occurs through the topological "Wess-Zumino" term $\int H$ only, which for closed membranes simply yields a phase factor proportional to the flux of $H$ through the 3-cycle [10]. This flux vanishes in the absence of a non-trivial topology in target space. Open membranes are charged under $H$ - however they only exist in the presence of five-branes on which they can end 22, 3 38]. The field $H$ is therefore the self-dual field strength of the two-form gauge field living on the five-brane worldvolume, plus the selfdual part of the bulk three-form gauge field $C$ for gauge invariance. Due to the non-linear self-duality constraint on $H$, one may worry whether a large $H$ limit exists. As discussed in [11], a generic constant non-linear self-dual three form $H$ may be written in a particular Lorentz frame as (a) $H=\tanh \phi d x^{045}+\sinh \phi d x^{123}$, so that there is a limit in which the magnetic component $H_{123}$ becomes very large while the electric component $H_{045}$ saturates to its critical value [16]. Even though the motions in the planes 123 and 045 do not decouple due to the membrane non-linearities, it may be useful to understand the effects of the large $H_{123}$ and of the critical $H_{045}$ fields separately. There also exists a non-generic "lightlike" case (b) $H=e^{\phi}\left(d x^{0}-d x^{1}\right)\left(d x^{23}+d x^{45}\right)$, where all components become large as $\phi \rightarrow \infty$, although $H^{2}=0$ throughout 17. With these preliminaries in mind, the action for an open membrane in the strong $H$ limit reduces to, after integrating by part,

$$
S=\int_{\Sigma} H_{i j k} X^{i} d X^{j} \wedge d X^{k}
$$

where $\Sigma$ is the membrane boundary, which we shall take connected for simplicity, and $X^{i}$ are the embedding coordinates of the membrane, fonctions of the worldsheet coordinates $(\sigma, \tau)\left(X^{1,2,3}\right.$ for case $(\mathrm{a})$, or $X^{0}-X^{1}, X^{2,3,4,5}$ in case (b), since the coordinate $X^{0}+X^{1}$ decouples). As noticed in 14, this is also the action for a vortex line in an incompressible and inviscid 3D fluid such as superfluid He II (after adding in the kinetic energy of the fluid, $\int v^{2} d^{3} x$ where $v$ is the velocity field of the fluid with vorticity localized along the line) [18. This action is symmetric under general diffeomorphisms on the worldsheet $\sigma$, and under diffeomorphisms preserving the three-form $H$ in target space - we'll refer to the latter as to volume-preserving diffeomorphisms (vpd), or selfdual volume-preserving diffeomorphisms (svpd) for case (b). Our hope is that despite the strong non-linearity of (1), these symmetries will allow to solve for the quantum theory associated to it. This hope is perhaps not unwarranted, in light of the existence of toy models with a cubic action which are nonetheless free 19] (see 20 for an application of these models to the quantum BPS membrane). The theory (11) has already been considered from a canonical 11, 12 or Batalin-Vilkovilsky 13] 39] point of view, however our approach will be rather differ- ent, and, as in [14], will emphasize the symmetry under vpd.

Classical dynamics. The equations of motion $H_{i j k} d X^{j} \wedge d X^{k}=0$ following from (11) imply that the embedding coordinates $X^{i}$ are fonctions of a single combination $f(\sigma, \tau)$ of the worldsheet coordinates. Up to reparameterisation of the worldsheet, one may choose $f=\sigma-\tau$, which shows that we are dealing with a chiral theory. The correlators of vertex operators such as $e^{i k X}(\sigma, \tau)$ will therefore be holomorphic in $z=\sigma-\tau$, with possible monodromies in the $z$ plane. More conveniently, we may choose $f=\sigma$, and think of $\tau$ as the time coordinate: in the absence of sources, the solutions of the equations of motion are therefore static loops $\gamma$ of arbitrary shape in target space. This is analogous to the statement that charged particles in two dimensions are frozen at a point in the presence of a strong magnetic field. The staticity is also a consequence of the invariance by time reparameterization, which implies a vanishing Hamiltonian. The canonical treatment of this system in case (a) was carried out long ago in the context of vortex lines in superfluids [21], and rediscovered many times since then. Since the time derivative appears in first order, the phase space is restricted by the primary constraints $C_{i}=\pi_{i}-H_{i j k} X^{j} \partial_{\sigma} X^{k}=0$ 40. The total momentum $\Pi_{i}=\int d \sigma \pi_{i}=H_{i j k} \int_{S} d X^{j} d X^{k}$ is therefore equal to the flux of $H$ through the surface $S$ bounded by the loop in target space. As in the case of dipoles in a magnetic field, the size of the membrane increases proportional to its linear momentum. Of the three constraints $C_{i}$, the two orthogonal to $\partial_{\sigma} X^{i}$ are second class, while $C=C_{i} \partial_{\sigma} X^{i}$ is first class, being the generator of the spatial diffeomorphisms. One may treat all constraints as second class (i.e. restricting the phase space of the system) by introducing a fourth constraint $C_{0}=0$ fixing the spatial diffeomorphisms, such as $C_{0}=X^{3}-g(\sigma)$ 41. Neglecting global issues, one may set $g(\sigma)=\sigma$, and obtain the canonical Dirac brackets

$$
\left[X^{1}(\sigma), X^{2}\left(\sigma^{\prime}\right)\right]=\delta\left(\sigma-\sigma^{\prime}\right) .
$$

The embedding coordinates are therefore pointwise noncommutative, hence the name "non-commutative string" given to the topological open membrane by the authors of [11, 12, 15]. One may however legitimately worry that all the interesting part of the dynamics lies in the global issues. Alternatively, one may consider reparameterisation invariant observables only. Those take the form $J(A)=\int_{\gamma} A_{i}\left[d X^{i} / d \sigma\right] d \sigma$ where $A_{i}$ is a one-form gauge field in target space. Upon coupling to $J(A)$ at time $\tau=0$, the equations of motion become $H_{i j k} \partial_{\sigma} X^{j} \partial_{\tau} X^{k}=$ $F_{i j} \partial_{\sigma} X^{j} \delta(\tau)$ where $F_{i j}=\partial_{i} A_{j}-\partial_{j} A_{i}$ is the curvature of $A$. For $d=3$, where $H_{i j k}=H \epsilon_{i j k}$, this implies that $\left[X^{i}\right]_{0^{-}}^{0^{+}}=\frac{1}{2} \epsilon^{i j k} F_{j k} / H$, meaning that the loop has moved 
by the action of the diffeomorphism generated by the vector $\xi=* d A / H$. It is essential to recognize that this diffeomorphism is divergenceless, $d * \xi=0$, i.e. it preserves the volume form $H$. Indeed the Dirac bracket of the operators $J(A)$ may be readily computed, and reads

$$
\left[J(A), J\left(A^{\prime}\right)\right]=J\left(A^{\prime \prime}\right)
$$

where $A^{\prime \prime}$ is the vector potential associated to the volumepreserving diffeomorphism generated by the Lie bracket $\left[\xi, \xi^{\prime}\right]$ [21]. The open topological membrane therefore classically furnishes a representation of the algebra of volume-preserving diffeomorphisms.

Quantisation. In order to understand the structure of the Hilbert space of the topological membrane, let us go back to the case of a charged particle in the limit of a strong magnetic field. The classical trajectories consist of Larmor orbits of vanishing radius as the magnetic field goes to infinity. Quantum mechanically, only the lowest Landau level remains, corresponding to a Gaussian wave packet around the origin, with infinite degeneracy (in unbounded geometry) arising from the action of areapreserving diffeomorphisms moving the origin around. In the present case, the classical configurations are now static loops, which can be moved and deformed from one into another under the action of the volume-preserving diffeomorphisms, so long as the topological invariants of the loop are preserved. One may thus foresee an Hilbert space consisting of all topological types of knots, with a degeneracy generated by the group of vpd, together with harmonic degrees of freedom around each loop, of infinite energy in the limit under consideration. The quantisation of the topological membrane thus amounts to the quantisation of the group of vpd (this point of view is also stressed in [14). Unfortunately, the quantisation based on the canonical commutation rules (2) leads to singularities in the correlators $\left\langle\prod_{i} J\left(\zeta_{i} e^{i k_{i} X^{i}}\right)\right\rangle$ whose proper treatment is rather unclear. In addition, the quantisation of the group of vpd stricto sensu is known not to exist: the cohomology group $H_{2}(G, G)$ classifying deformations at leading order is non-trivial, but the corresponding deformation is obstructed at second order [23]. It is conceivable however that the structure to be deformed is not precisely the group of vpd, but a structure equivalent to it in the classical regime, as we now discuss.

Vpd and Nambu dynamics. We have seen previously that a volume-preserving diffeomorphism in 3 dimensions can be written as the divergence of a one-form, $\xi=* d A$. By a slight extension of the Darboux theorem, it is also possible to locally represent the two-form $* \xi$ as a product $d H \wedge d K$, where $(H, K)$ are a pair of symplectic local coordinates. The diffeomorphism $\xi$ then acts on fonctions on the three-dimensional "phase space" $\left(x^{1}, x^{2}, x^{3}\right)$ through the Nambu bracket [24,

$$
\delta_{\xi} F=\{H, K, F\}:=\epsilon^{i j k} \partial_{i} H \partial_{j} K \partial_{k} F
$$

analogous to the Poisson bracket for area-preserving diffeomorphisms. This bracket is a completely antisymmetric linear fonction of its arguments, and satisfies the derivation property

$$
\left\{f_{1} f_{2}, f_{3}, f_{4}\right\}=f_{1}\left\{f_{2}, f_{3}, f_{4}\right\}+\left\{f_{1}, f_{3}, f_{4}\right\} f_{2}
$$

and the so called Fondamental Identity [25]

$$
\begin{aligned}
& \left\{\left\{f_{1}, f_{2}, f_{3}\right\}, f_{4}, f_{5}\right\}+\left\{f_{3},\left\{f_{1}, f_{2}, f_{4}\right\}, f_{5}\right\} \\
+ & \left\{f_{3}, f_{4},\left\{f_{1}, f_{2}, f_{5}\right\}\right\}=\left\{f_{1}, f_{2},\left\{f_{3}, f_{4}, f_{5}\right\}\right\}
\end{aligned}
$$

generalizing the Jacobi identity of the Poisson bracket. As is well known, this structure was introduced by Nambu as an alternative way to construct dynamical systems satisfying the Liouville property, ie the conservation of probability on phase space [24] (see [26] for an application to the quantization of strings). Such systems satisfy the evolution equation

$$
d F / d \tau=\{H, K, F\}
$$

for a pair of fixed generalized Hamiltonians $(H, K)$ and $F$ a function on phase space. Interestingly, these equations of motion follow from an action functional 25] 42]

$$
S=\int d \sigma d \tau\left(\epsilon_{i j k} X^{i} \partial_{\sigma} X^{j} \partial_{\tau} X^{k}-H d K d \tau\right)
$$

analogous to the action $S=\int p d q-H d t$ in Hamiltonian dynamics, which in the case of vanishing generalized Hamiltonians $H=K=0$ is precisely our topological membrane action (1D). The quantisation of the topological membrane is therefore equivalent to the quantisation of Nambu dynamics. In particular, one would like to find an analogue of geometric quantisation in which the Nambu bracket is deformed while preserving its fundamental properties - due to the ambiguity in the choice of Hamiltonians $(H, K)$ for a given diffeomorphism $\xi$, this question is not strictly equivalent to the quantisation of vpd, so that the no go theorem in [23] may hopefully be evaded. An number of attempts at this problem have been made [25, 27, 28, 29], but those constructions, with the possible exception of the one based on Zariski quantisation [30], have to drop some of the requirements imposed on the Nambu bracket, and seem rather ad hoc. It is a challenge to derive them from a proper quantisation of the topological open membrane.

Clebsch parameterisation and Chern-Simons theory. In the previous section, we have argued that a divergenceless field in 3 dimensions can be parameterized either by a gauge field, $\xi=* d A$, or by a pair of scalar 
fields up to canonical transformation, $\xi=* d H \wedge d K$. This implies that $A-H d K$ is closed, so that any gauge field $A$ can be represented locally in terms of three scalar fields $(L, H, K)$ as $A=d L+H d K$. This is a familiar representation in incompressible hydrodynamics, known as the Clebsch parameterisation of the velocity field $\xi$ (see e.g. [31] for a discussion of this representation). The Chern-Simons invariant $I=\int A \wedge d A$ of the Abelian gauge field $A$ is known as the total helicity, or asymptotic Hopf invariant 32, and is conserved in a perfect fluid. Expressed in terms of the Clebsch parameterisation, it becomes $I=\int d L \wedge d H \wedge d K$, our familiar membrane action again! This seems to indicate that the quantum membrane is equivalent to Abelian Chern-Simons on a manifold with a boundary, or yet equivalently a $U(1)$ WZW model, on the boundary, i.e. a free chiral scalar field in two dimensions 43. Unfortunately, the map $(L, H, K) \leftrightarrow A$ is very singular, the $S O(3)$ symmetry is non-linearly realized in the gauge field parameterization and the Jacobian it induces may have a non-trivial effect. On the other hand, the Chern-Simons theory may be a sensible definition of an otherwise ill-defined topological quantum membrane theory.

Vpd and Nambu dynamics in higher dimensions. Our discussion of volume-preserving diffeomorphisms and Nambu dynamics has so far been mostly restricted to the three-dimensional case (a). However the topological membrane action (11) in principle makes sense in any dimension, and it is useful to recall some features of three-form preserving diffeomorphisms in dimension $D>3$. Firstly, note that a closed three-form $H$ has $(D-1)(D-2) / 2$ degrees of freedom; in contrast to the symplectic case, for $D>4$ it can therefore not be brought into a constant form by a diffeomorphism (however a selfdual three-form in $D=5+1$ has only 5 degrees of freedom so can be made constant by a diffeomorphism). Second, assuming $H$ to be constant, for $D>5$ it still cannot be put into a standard form, but still carries moduli under the group of linear diffeomorphisms $G l(D, R)$ (for $D \leq 5$, it can be dualized into a form of lower degree and a standard form exists). Third, for a given constant three-form $H_{i j k}$, three-form-preserving diffeomorphisms $\xi^{i}=\zeta^{i} e^{i p x}$ have to satisfy the relation $H_{i[j k} p_{l]} \zeta^{i}=0$ (i) for all values of the indices $(j k l)$. In general, such a polarization $\zeta$ only exists for particular momenta $p$ (except again in $D \leq 5$, where $H$ can be dualized into a form of lower degree). For special choices of $H$ however, satisfying the quadratic equation $H_{i j[k} H_{l m] p}=0$ (ii) resulting from the elimination of $\zeta^{i}$ for all $p^{i}$ in (i), there is no constraint on $p$ anymore, and one can have arbitrary momenta. This is in particular the case when $H$ degenerates to a single monomial such as in case (a) above. This is also true in case (b), where any diffeomorphism $f\left(x_{i}\right)\left(\partial_{0}+\partial_{1}\right)$ preserves the form $H=\left(d x^{0}-d x^{1}\right)\left(d x^{23}+d x^{45}\right)$ (of course, any area-preserving diffeomorphisms in the (2345) would also preserve $H$ ). Conversely, we could try to define a Nambu bracket in higher dimension as $\{H, K, F\}=$ $\theta^{i j k} \partial_{i} F \partial_{j} K \partial_{k} F$ where $\theta^{i j k} \partial_{i} \wedge \partial_{j} \wedge \partial_{k}$ is a three-vector. This definition automatically satisfies the antisymmetry and derivation property. However the Fundamental Identity (6) is only satisfied under special conditions, which in the case of constant $\theta$ reduce to the algebraic condition $\theta^{i j[k} \theta^{l m n]}=\theta^{k j[i} \theta^{l m n]}$ (iii) 25]. This includes the degenerate case (a) $\theta=\partial_{123}$ but excludes non decomposable tensors such as $\theta=\partial_{123}+\partial_{456}+\ldots$ (in other words, Nambu dynamics lack extensivity). In particular, it excludes the lightlike case (b) of interest for the noncommutative five-brane. It would be interesting to see if the conditions (ii) or (iii) could be deduced from the quantum consistency of the membrane action (1), and if they bear any relation to the non-linear self-duality of the 3 -form field strength $H$ on the five-brane worldvolume.

Vpd and non-Abelian two-form dynamics. Despite providing some geometrical insight, the observations we have presented so far have produced few results on the problem of the quantum topological membrane. In the last part of this note, we will propose a non-Abelian deformation of the dynamics of a two-form, based on the ideas developped before. Our approach is a simple generalisation of an argument by Susskind and Bahcall in the context of the Quantum Hall effect, relating the dynamics of a perfect fluid in two dimensions to a gauge theory for the group of area-preserving diffeomorphisms 34]. Following on their steps, we consider a perfect fluid in $3+1$ dimensions in comoving (Lagrange) coordinates,

$$
\mathcal{L}=\int d^{3} x d \tau\left[\frac{1}{2}\left(\frac{d y^{i}}{d t}\right)^{2}-V\left(\left|\operatorname{det} \frac{\partial x}{\partial y}\right|\right)\right]
$$

where $y^{i}(x, t)$ is the position of the fluid particle labelled by its position at $x^{i}$ at $t=0$, where we assume a constant density normalized to 1 and also set the mass to 1 . The potential $V$ describes short-range forces, and is assumed to depend on the local density $\rho=|\operatorname{det}(\partial x / \partial y)|$ at time $t$, with a stable minimum at $\rho=1$. By construction, this Lagrangian is invariant under volume preserving diffeomorphisms of the labelling coordinate $x^{i}$. Now we consider small perturbations around equilibrium at $\rho=1$, i.e. sound waves propagating in this perfect fluid. We parameterize the fluctuations by a twoform, $y^{i}(x, t)=x^{i}+\epsilon^{i j k} b_{j k}(x, t)$. Under a volumepreserving infinitesimal diffeomorphism $\xi$, the field $y^{i}$ changes by $\xi^{j}(x, t) \partial_{j} y^{i}$. Parameterizing $\xi$ by a one-form $\xi^{i}=\epsilon^{i j k} \partial_{i} a_{j}$, we find that the two-form $b$ transforms as

$$
\delta_{\xi} b_{i j}=\partial_{i} a_{j}-\partial_{j} a_{i}+\left\{a, b_{i j}\right\}
$$

where we employed the bracket $\{a, f\}:=\epsilon^{k l m} \partial_{l} a_{m} \partial_{k} f$. Equivalently, we may have parameterized $\xi$ by a pair of 
scalar fields $\xi^{i}=\epsilon^{i j k} \partial_{j} H \partial_{k} K$, and expressed the variation of the two-form $b$ as

$$
\delta_{\xi} b_{i j}=\partial_{i} H \partial_{j} K-\partial_{j} H \partial_{i} K+\left\{H, K, b_{i j}\right\}
$$

where the last term is the Nambu bracket (4). At leading order in $b$, we find the usual $\delta b=d a$ gauge variation of an Abelian two-form, however the transformation receives a correction linear in $b$, analogous to the Yang-Mills variation for non-Abelian or non-commutative one-form gauge fields. A gauge invariant field strength can be readily constructed by considering the Jacobian $h_{123}:=\partial y / \partial x-1=$ $1 / \rho-1$, by construction covariant under vpd. In terms of the two-form $b$, this is

$$
\begin{aligned}
& h_{123}=\left|\begin{array}{ccc}
1+\partial_{1} b_{23} & \partial_{2} b_{23} & \partial_{3} b_{23} \\
\partial_{1} b_{31} & 1+\partial_{2} b_{31} & \partial_{3} b_{31} \\
\partial_{1} b_{12} & \partial_{2} b_{12} & 1+\partial_{3} b_{12}
\end{array}\right|-1 \\
&= {\left[\partial_{1} b_{23}+\text { circ }\right]+\left[\left\{b_{12}, b_{13}\right\}_{23}+\text { circ }\right] } \\
&+\left\{b_{12}, b_{23}, b_{31}\right\}
\end{aligned}
$$

The leading term is indeed the usual exterior derivative $h=d b+\ldots$, but there are now up to cubic terms in $b$, which ensure the gauge covariance $\delta_{\xi} h_{i j k}=$ $\left\{a, h_{i j k}\right\}=\left\{H, K, h_{i j k}\right\}$ under vpd. In the above expression, $\{f, g\}_{i j}$ denotes the Poisson bracket in the plane $(i j)$, i.e. $\{f, g\}_{i j}=\partial_{i} f \partial_{j} g-\partial_{j} f \partial_{i} g$.

Expanding the potential $V(\rho)$ to second order around its minimum at $\rho=1$, we obtain for the Lagrangian for the sound waves described by the two-form $b$,

$$
\mathcal{L}=\int d^{3} x d \tau \frac{1}{2 \cdot 2 !}\left(\partial_{\tau} b_{i j}\right)^{2}-\frac{1}{2 \cdot 3 !} h_{i j k}^{2} .
$$

In addition, one should impose the Gauss constraint restricting the system to the gauge invariant sector. The conserved charge resulting from invariance under vpd reads

$$
\notin=\int d^{3} x\left[\partial_{i} a_{j}-\partial_{j} a_{i}+\epsilon^{k l m} \partial_{l} a_{m} \partial_{k} b_{i j}\right] \partial_{t} b_{i j} \quad(14)
$$

which vanishes for all gauge parameters $a_{i}$ under the Gauss constraint

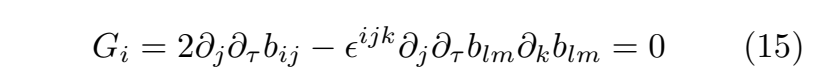

This constraint can be enforced by means of a Lagrange multiplier $b_{0 i}$, so that the Lagrangian takes the apparently Lorentz covariant form $\mathcal{L}=\int d^{3} x d \tau h_{\mu \nu \rho}^{2}$ with

$$
h_{0 i j}=\partial_{0} b_{i j}+\partial_{j} b_{0 i}+\partial_{i} b_{j 0}+\epsilon^{k l m} \partial_{k} b_{0 l} \partial_{m} b_{i j}
$$

Unfortunately, one can show that there is no choice of the gauge variation $\delta_{\xi} b_{0 i}$ that renders $h_{0 i j}$ gauge covariant, so that the free Lagrangian needs to be supplemented by a possibly infinite sum of terms dependent on $b_{0 i}$. In the temporal gauge $b_{0 i}=0$ however, the theory based on the action (13) supplemented by the Gauss constraint
(15) is perfectly consistent, and yields a non-Abelian deformation of the dynamics of a two-form in $3+1$ dimensions, based on the group of volume preserving diffeomorphisms 44]. It would be interesting to see if the algebraic structure of (10), (11), (12) can be abstracted, and the group of vpd replaced by other groups such as finite Lie groups. It is also important to generalize it to $5+1$ dimensions, if one is to make contact with the five-brane. As a possible step in this direction, and prompted by the hydrodynamical picture advocated in [36], one may consider a fluid of strings rather than particles: the same formulae as above still hold, but for adding a fifth worldsheet coordinate $\sigma$, the missing sixth coordinate being presumably due to the decoupling of the $X^{0}-X^{1}$ coordinate in (1). The effect of a background three-form would then be to add a coupling $\int d^{3} x d \tau d \sigma B_{12} \partial_{\sigma} B_{23} \partial_{\tau} B_{31}$ analogous to the Chern-Simons term in the Quantum Hall effect. It will be exciting to see whether M-theory or one of its avatars, after having tamed the quantum Hall effect, can also master the quantum vortex in superfluid He II.

Acknowledgments. These observations grew out of initial discussions with S. Minwalla and R. Gopakumar whom I gratefully acknowledge but who should not be blamed on any misconception herein. They were presented in May 2001 at the "Avatars of M-theory" program at ITP, Santa Barbara, whom I wish to thank for the kind hospitality. I also benefited from discussions with D. Berman, C. Hofman, D. Minic, Y. Matsuo, M. van Raamsdonk, S. da Silva and A. Strominger, and I am grateful to C. Roger for communicating his results.

* Electronic address: pioline@lpthe.jussieu.fr

[1] C. G. Callan, J. A. Harvey and A. Strominger, Nucl. Phys. B 367, 60 (1991).

[2] E. Witten, Nucl. Phys. B 443, 85 (1995) arXiv:hepth/9503124; A. Strominger, Phys. Lett. B 383, 44 (1996) arXiv:hep-th/9512059.

[3] K. Ezawa, Y. Matsuo and K. Murakami, Phys. Rev. D 57, 5118 (1998) arXiv:hep-th/9707200; P. Brax and J. Mourad, Phys. Lett. B 416, 295 (1998) arXiv:hepth/9707246; C. S. Chu and E. Sezgin, JHEP 9712, 001 (1997) arXiv:hep-th/9710223.

[4] A. Dasgupta, H. Nicolai and J. Plefka, arXiv:hepth/0201182

[5] X. Bekaert, M. Henneaux and A. Sevrin, Phys. Lett. B 468, 228 (1999) arXiv:hep-th/9909094.

[6] I. R. Klebanov and A. A. Tseytlin, Nucl. Phys. B 475, 164 (1996) arXiv:hep-th/9604089; J. A. Harvey, R. Minasian and G. W. Moore, JHEP 9809, 004 (1998) arXiv:hep-th/9808060; M. Henningson and K. Skenderis, JHEP 9807, 023 (1998) arXiv:hep-th/9806087;

[7] A. Connes, M. R. Douglas and A. Schwarz, JHEP 9802, 003 (1998) arXiv:hep-th/9711162.

[8] N. Seiberg and E. Witten, JHEP 9909, 032 (1999) arXiv:hep-th/9908142.

[9] M. Aganagic, J. Park, C. Popescu and J. H. Schwarz, 
Nucl. Phys. B 496, 191 (1997) arXiv:hep-th/9701166: P. S. Howe, E. Sezgin and P. C. West, Phys. Lett. B 399, 49 (1997) arXiv:hep-th/9702008.

[10] E. Bergshoeff, E. Sezgin and P. K. Townsend, Phys. Lett. B 189, 75 (1987); E. Bergshoeff, E. Sezgin and P. K. Townsend, Annals Phys. 185, 330 (1988).

[11] E. Bergshoeff, D. S. Berman, J. P. van der Schaar and P. Sundell, Nucl. Phys. B 590, 173 (2000) hepth/0005026; E. Bergshoeff, D. S. Berman, J. P. van der Schaar and P. Sundell, Phys. Lett. B 492, 193 (2000) hep-th/0006112.

[12] S. Kawamoto and N. Sasakura, JHEP 0007, 014 (2000) arXiv:hep-th/0005123.

[13] J. Park, hep-th/0012141; C. M. Hofman and W. K. Ma, hep-th/0102201.

[14] Y. Matsuo and Y. Shibusa, JHEP0102, 006 (2001) hepth/0010040.

[15] A. K. Das, J. Maharana and A. Melikyan, JHEP 0104, 016 (2001) arXiv:hep-th/0103229.

[16] R. Gopakumar, S. Minwalla, N. Seiberg and A. Strominger, JHEP 0008, 008 (2000) arXiv:hep-th/0006062.

[17] O. Aharony, M. Berkooz and N. Seiberg, Adv. Theor. Math. Phys. 2, 119 (1998) arXiv:hep-th/9712117; O. Aharony, J. Gomis and T. Mehen, JHEP 0009, 023 (2000) [arXiv:hep-th/0006236]; M. Berkooz, arXiv:hepth/0010158.

[18] F. Lund and T. Regge, Phys. Rev. D 14, 1524 (1976).

[19] P. Etingof, D. Kazhdan, A. Polishchuk, math.AG/ 0003009 .

[20] D. Kazhdan, B. Pioline and A. Waldron, to appear in Comm. Math. Phys. arXiv:hep-th/0107222; B. Pioline, H. Nicolai, J. Plefka and A. Waldron, JHEP 0103, 036 (2001) arXiv:hep-th/0102123.

[21] M. Rasetti and T. Regge, Physica 80A (1975) 217; also in proceedings of "Group Theoretical Methods In Physics", Trieste 1983, 311.

[22] A. Zee, Nucl. Phys. B 421, 111 (1994).

[23] P. Lecomte and C. Roger, J. Diff. Geom. 44 (1996) 529.

[24] Y. Nambu, Phys. Rev. D 7, 2405 (1973).

[25] L. Takhtajan, Commun. Math. Phys. 160, 295 (1994) hep-th/9301111.

[26] Y. Nambu, Phys. Lett. B 92, 327 (1980).

[27] R. Chatterjee and L. Takhtajan, Lett. Math. Phys. 37, 475 (1996) hep-th/9507125.
[28] J. Hoppe, Helv. Phys. Acta70, 302 (1997) hepth/9602020.

[29] H. Awata, M. Li, D. Minic and T. Yoneya, JHEP0102, 013 (2001) hep-th/9906248.

[30] G. Dito, M. Flato, D. Sternheimer and L. Takhtajan, Commun. Math. Phys. 183, 1 (1997) hep-th/9602016.

[31] R. Jackiw, physics/0010042.

[32] V.I. Arnold, Sel. Math. Sov. 5 (1986) 327.

[33] I. Rudychev, JHEP 0104, 015 (2001) arXiv:hepth/0101039.

[34] S. Bahcall and L. Susskind, Int. J. Mod. Phys. B 5, 2735 (1991); L. Susskind, hep-th/0101029.

[35] D. Z. Freedman and P. K. Townsend, Nucl. Phys. B 177, 282 (1981).

[36] G. W. Gibbons and P. C. West, hep-th/0011149.

[37] Due to the self-duality condition we avoid mentioning the action and focus on the equations of motion only.

[38] Membranes could also end on Horava-Witten 9-branes, or on one-dimensional defects (see e.g. 44).

[39] It may be useful to develop a BV quantization of the two-dimensional theory (11) itself, instead of the threedimensional theory with boundaries considered in [13. For that purpose, the equation $* d X^{i}=H_{i j k} X^{j} d X^{k}$ is a very natural gauge-fixing function, and it would be interesting to study the field configurations onto which it localizes.

[40] Of course, these constraints would be relaxed by keeping the kinetic term in the action. The stringy choice $\int d \sigma d \tau\left(\partial_{\tau} X^{i}\right)^{2}-\left(\partial_{\sigma} X^{i}\right)^{2}$ made by Regge and Lund 18] does not seem adequate for the membrane case at hand, however it does lead to interesting soliton solutions [22].

[41] Another interesting choice is $C_{0}=\sum\left(X^{i}\right)^{2}-g(\sigma)$, which yields the commutation rules for $S U(2)$ currents at level 0 (S. Minwalla, private communication).

[42] The Euler-Lagrange equations following from this action are $\epsilon_{i j k}\left(\partial X^{j} / \partial \tau-\left\{H, K, X^{j}\right\}\right) \partial X^{k} / \partial \sigma=0$, which imply (đ) for $F=X^{i}$ after using the symmetry under spatial diffeomorphisms.

[43] A somewhat similar equivalence to $S U(2)$ WZW model with the topological term only has been produced in 33 .

[44] It should be noted that this deformation is different from the Freedman-Townsend vertex $\operatorname{Tr}(b \wedge[* d b, * d b])$, which still yields an Abelian gauge symmetry on-shell [35]. 